\title{
Psicoterapia Pela Internet: Viável ou Inviável?
}

Online Therapy: Viable or Unviable?

Psicoterapia a Través de Internet: ¿Viable o Inviable?

Maria Adélia

Minghelli Pieta \& William B. Gomes

Universidade Federal do Rio Grande do Sul

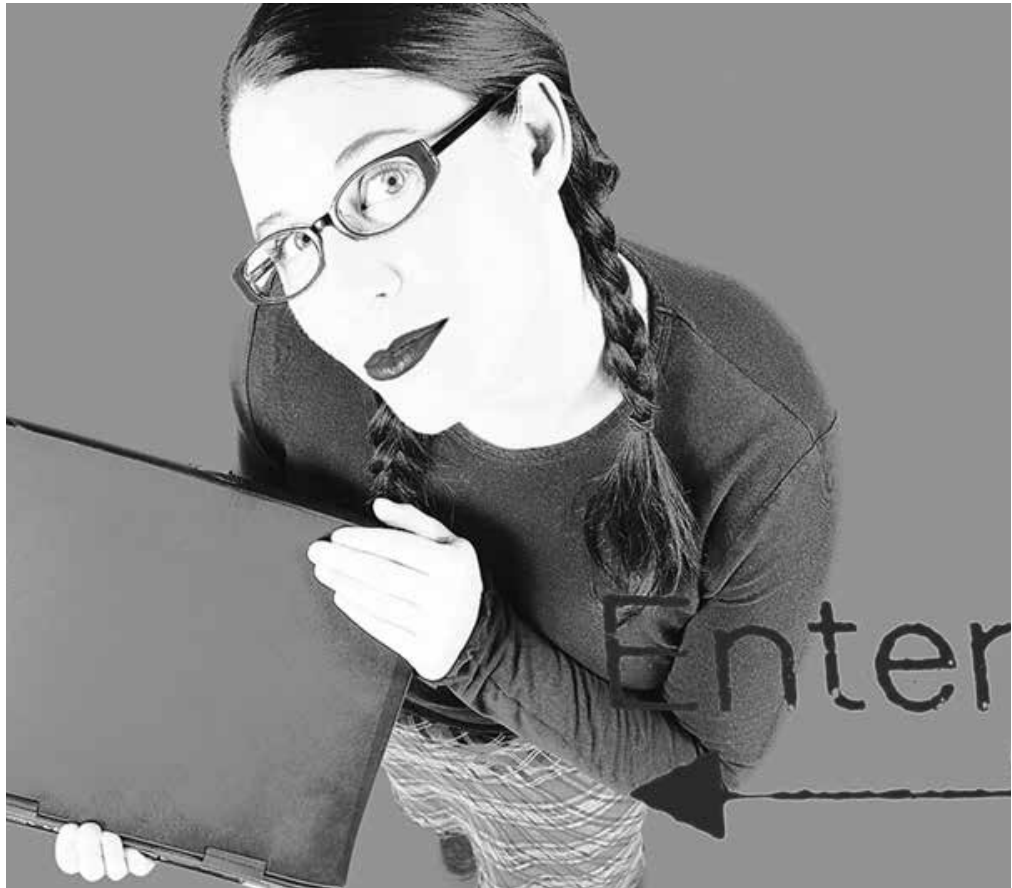


Resumo: A psicoterapia pela internet é uma prática que, no Brasil, só é permitida aos psicólogos na forma de pesquisa. O objetivo do presente artigo foi realizar uma revisão dos estudos em psicoterapia pela internet, discorrendo sobre os recursos e os limites desse atendimento psicológico e suas implicações para a relação terapêutica e para a efetividade do tratamento. São apresentadas questões legais e éticas concernentes à prática. Os resultados apontaram similaridades entre a relação terapêutica online e a presencial, mostrando-se a psicoterapia pela internet efetiva nas mais distintas modalidades, embora a maioria dos estudos seja sobre intervenções cognitivo-comportamentais. As questões legais e éticas podem ser parcialmente solucionadas com diretrizes claras das organizações profissionais e com o treino de terapeutas na modalidade online. Concluímos que a psicoterapia pela internet, embora requeira maiores estudos, anuncia-se como uma prática viável e promissora.

Palavras-chave: Psicoterapia. Internet. Avaliação terapêutica. Terapia online.

Abstract: The practice of online therapy is only allowed to psychologists in Brazil for research. The aim of this paper was to review studies on online therapy, discussing the features and limits of this type of psychological treatment and its implications for the therapeutic relationship and treatment effectiveness. Legal and ethical issues concerning the practice are presented. The results pointed to similarities between the therapeutic relationship online and face-to-face therapy, showing that online therapy is effective in its different modalities, although most studies deal with cognitive-behavioral interventions. Legal and ethical issues can be partially solved with clear guidelines of professional organizations and training of therapists in online mode. We conclude that online therapy requires more study, but already presents itself as a viable and promising practice.

Keywords: Psychotherapy. Internet. Treatment effectiveness evaluation. Online therapy.

Resumen: La psicoterapia a través de Internet en Brasil sólo está permitida a los psicólogos en la forma de investigación. El objetivo de este trabajo fue revisar los estudios de la psicoterapia a través de Internet, hablando de las características y los límites de esto tratamiento psicológico y sus implicaciones para la relación terapéutica y la efectividad del tratamiento. Se muestran problemas legales y éticos relacionados con la práctica. Los resultados apuntaron a las similitudes entre la relación terapéutica online y cara a cara, muestrando que la psicoterapia a través de Internet es efectiva en sus más distintas modalidades, aunque la mayoría de los estudios se trata de intervenciones cognitivo-conductuales. Las cuestiones jurídicas y éticas pueden ser parcialmente resueltas con claras diretrices de las organizaciones profesionales y formación de los terapeutas en la modalidade online. Concluimos que la psicoterapia a través de Internet, aunque requiere más estudio, se anuncia como una práctica viable y prometedora.

Palabras clave: Psicoterapia. Internet. Evaluación terapêutica. Terapia online.

A psicoterapia pela internet é uma prática difundida no exterior e que tem apresentado resultados benéficos. No Brasil, essa modalidade terapêutica só é permitida pelo Conselho Federal de Psicologia (CFP) na forma de pesquisa (Resolução CFP no012/2005), o que pode ser interpretado como um incentivo ao seu estudo. O tema tem sido foco de eventos recentes, como o Seminário de Serviços Psicológicos Online, do CFP, de 2011, e o Seminário Psicologia Online São Paulo 2011, do Conselho Regional de Psicologia de São Paulo. Essa mobilização deve-se à necessidade de se reavaliar o campo de atuação dos psicólogos frente às transformações da era digital. Em vista de tais considerações, o objetivo do presente artigo foi realizar uma revisão dos estudos empíricos de psicoterapia pela internet, discorrendo sobre os recursos e os limites desse atendimento psicológico e sobre suas implicações para a relação terapêutica e efetividade do tratamento. Desse modo, a presente exposição está organizada em quatro partes. Na primeira, discorre sobre o início e o desenvolvimento da psicoterapia pela internet, com atenção à terminologia empregada e à distinção entre psicoterapia online e intervenções baseadas na internet. A segunda volta-se para o núcleo central dos tratamentos psicológicos e quer saber o que se altera ou como se configura a relação terapêutica nesse novo ambiente. A terceira apresenta e analisa evidências empíricas sobre a efetividade da psicoterapia pela internet, em estudos quantitativos e qualitativos recentes. A exposição examina, por fim, as questões legais e éticas concernentes ao assunto. 
Esperamos elucidar alguns aspectos referentes à prática e agregar novos elementos ao debate que se estabelece sobre o tema.

\section{Atendimento psicoterápico pela internet: história, conceituação e prática}

Apesar de o atendimento psicoterápico por telefone ser uma prática que remonta à década de 50 (Godleski, Nieves, Darkins, \& Lehmann, 2008; Scharff, 2012), a psicoterapia pela internet, em seu início, há mais de uma década, recebeu grande oposição. Acreditava-se que a relação terapêutica ficaria comprometida por um empobrecimento da comunicação não verbal, elemento considerado essencial à interação entre terapeuta e paciente. Preocupações éticas acerca da confidencialidade, da identidade de pacientes e terapeutas, dos papéis desempenhados na rede e do manejo de situações de emergência tomavam grande relevância. Questões legais referentes à jurisdição e ao regulamento da prática também inquietavam, bem como considerações sobre a necessidade de treino de terapeutas na nova modalidade psicoterápica (Barak, Hen, Boniel-Nissim, \& Shapira, 2008).

No entanto, as apreensões diminuíram diante do alcance oferecido pela nova mídia e das tentativas pioneiras bem-sucedidas (Proudfoot et al., 2011). Por conseguinte, um maior número de profissionais passou a realizar psicoterapia online, tecnologias avançadas facilitaram a prática, códigos de ética foram ajustados, e cursos de formação começaram a ser oferecidos. Por outro lado, os pacientes se mostraram receptivos a essa nova forma de intervenção terapêutica. Muita das perguntas acerca da efetividade da terapia online, se era tão efetiva quanto a terapia tradicional e de que forma diferentes métodos e variáveis associados à terapia pela internet influenciavam sua efetividade foram sendo respondidas por pesquisadores (Barak et al., 2008). Atualmente, há evidências a favor da terapia online nos países em que é praticada, como Austrália, Estados Unidos e Reino Unido. Pesquisas têm mostrado que a psicoterapia pela internet é efetiva, amplia o acesso à terapia e diminui seus custos (Proudfoot et al., 2011). Resultados positivos têm sido encontrados na utilização de terapias baseadas na internet para o tratamento da depressão (Kessler et al., 2009), ansiedade (Cuijpers et al., 2009), fobia (Titov et al., 2011), transtorno do pânico (Bergström et al., 2010), estresse pós-traumático (Klein et al., 2010) e transtornos alimentares (Carrad et al., 2011). Estudos recentes mostram que a terapia online também pode ser promissora no tratamento das adições e do jogo patológico (Gainsbury \& Blaszczynski, 2011), da psicose (Sharp, Kobak, \& Osman, 2011), de refugiados e imigrantes (Mucic, 2010), de depressão em pacientes terminais (Cluver, Schuyler, Frueh, Brescia, \& Arana, 2005) e de deficientes auditivos (Moore, Guthmann, Rogers, Frakeer, \& Embree, 2009). Não apenas adultos podem beneficiar-se da psicoterapia pela internet mas também adolescentes e crianças (March, Spence, \& Donovan, 2009; Spence et al., 2011). O anonimato pode auxiliar na busca de atendimento psicológico de pessoas introvertidas, com transtorno de ansiedade, como agorafobia e fobia social, com problemas de imagem corporal (Leibert, Archer Jr., Munson, \& York, 2006), bem como de adolescentes e usuários de substâncias (Gainsbury \& Blaszczynski, 2011; Hanley, 2009; King et al., 2006).

Apesar de oferecer diversas vantagens como disponibilidade, conveniência, acessibilidade, baixo custo, anonimato, privacidade e redução de estigma (Cartreine, Ahern, \& Locke, 2010), a psicoterapia pela internet requer maiores estudos para uma melhor compreensão de seus efeitos. Até o presente, a maior parte das pesquisas na área tem 
sido conduzida no exterior, sendo escassa a produção nacional. Investigações empíricas sobre essa modalidade terapêutica são praticamente inexistentes em nosso país, apesar do debate que se instaura acerca do tema.

Um problema encontrado por pesquisadores tem sido estabelecer diretrizes para as investigações em psicoterapia online. Há heterogeneidade nas pesquisas, na utilização de terminologias e definições, bem como são encontrados problemas metodológicos e de conclusões questionáveis de estudos (Proudfoot et al., 2011). A falta de consenso nas definições adotadas faz com que termos como web-based therapy, e-therapy, cybertherapy, eHealth, e-Interventions, computer-mediated interventions e online counseling sejam utilizados como sinônimos, quando designam práticas diferentes. Uma padronização da terminologia faz-se necessária para maior discriminação do campo (Barak, Klein, \& Proudfoot, 2009).

Há uma distinção clara entre psicoterapia online e intervenções baseadas na internet. Psicoterapia pela internet ou psicoterapia online refere-se, mais precisamente, às terapias realizadas pelo profissional por e-mail, chat, mensagem instantânea (MSN), áudio ou videoconferência, sem excluir a possibilidade de encontros presenciais. Intervenções baseadas na internet, por sua vez, são programas computadorizados de terapia, com ou sem participação de terapeuta, que incluem CDs, DVDs, CDROMS, realidade virtual, aplicativos de celulares, tarefas e feedback online. Esses programas são prescritos por profissionais de saúde ou são oferecidos em websites, com o objetivo de auxiliar no tratamento e na prevenção de transtornos mentais e recaída, e buscam criar mudanças positivas e melhorar os conhecimentos em saúde mental por meio de material informativo, de fóruns moderados e de outros dispositivos da web. Um exemplo é uma intervenção que inclua textos e gráficos para transmitir informação, instruções de áudio para relaxamento progressivo, vídeo apresentando casos, suporte do terapeuta por e-mail, bem como envio automático de e-mails e/ou lembretes por SMS (Proudfoot et al., 2011).

Os programas computadorizados de terapia são excelentes paliativos para filas de espera (Proudfoot et al., 2011). Indivíduos que aguardam atendimento podem executálos para aprender a lidar com sintomas depressivos ou de ansiedade até que recebam tratamento apropriado. Seu caráter preventivo faz parte de uma tendência de pesquisa e de desenvolvimento que tem crescido muito na Austrália, a da saúde eletrônica preventiva (preventive eHealth).

Indivíduos que vivem em regiões remotas e não têm acesso a uma terapia também podem beneficiar-se das intervenções baseadas na internet. Um exemplo foi a oferta do programa FearFighter para pânico e fobia a habitantes de zonas rurais da Escócia. Os pacientes adquiriram senha para acessá-lo no posto de saúde e executaram-no de casa durante dez semanas, obtendo apoio de terapeuta por telefone se necessário. Uma vez por semana, um terapeuta ligava para os pacientes para conferir seu estado. Ao cabo do tratamento, os pacientes relataram sentirem-se melhor do que antes (MacGregor, Hayward, Peck, \& Wilkes, 2009).

As intervenções baseadas na internet podem ser adaptadas ao usuário, rastreando seu progresso e provendo feedback, ou padronizadas e oferecidas a grandes populações. Nos programas em que há interação com o terapeuta, esta se dá por e-mail, videoconferência, chat, MSN, telefone e mesmo encontros presenciais. Os programas que envolvem mínimo apoio do terapeuta são chamados de autoajuda monitorada (guided self-help), e são suficientes para alguns 
pacientes que, ao cabo do processo, decidem não precisar de mais terapia (Macdonald, Mead, Bower, Richard, \& Lovell, 2007). As intervenções baseadas na internet que não envolvem nenhuma interação com terapeuta, as terapias automonitoradas, são oferecidas de duas formas: programas que precisam de registro, triagem e senha para serem acessados (e.g. Beating the Blues) e programas acessados diretamente em websites (e.g. MoodGym). Alguns desses programas não exigem conhecimento prévio de internet.

As terapias online e intervenções baseadas na internet variam em seu sincronismo, e podem envolver comunicação sincrônica, isto é, imediata (e.g. chat) ou assincrônica, quer dizer, com algum atraso (e.g. e-mail), que pode ser curto (e.g. e-mail diário) ou longo (e.g. e-mail de resposta do terapeuta a cada três dias). Na psicoterapia online via e-mail, o terapeuta deve combinar intervalos determinados de resposta para não suscitar ansiedade no paciente (Rochlen, Zack, \& Speyer, 2004). Nas intervenções baseadas na internet, o sincronismo tanto pode ser predeterminado pelo programa como adaptado às necessidades do paciente. Também o tempo de duração entre as sessões varia. Enquanto alguns programas prescrevem sessões semanais ou diárias, outros não determinam o número de sessões, permitindo acesso não estruturado por um determinado período de tempo. Há programas bastante variáveis, como, por exemplo, que iniciam com uma alta frequência de sessões e diminuem a frequência após um ponto crítico. Igualmente, o feedback varia em termos de quantidade, frequência e rapidez de resposta. O feedback humano tende a ser mais adaptado às circunstâncias do paciente em comparação com o automático (Barak et al., 2009).

Uma das vantagens da utilização de tecnologias eletrônicas e digitais em tratamentos psicoterápicos é que o indivíduo pode acessá-las do ambiente em que se encontra e no momento em que sente ser adequado. Esse dispositivo terapêutico é ecológico, pois adapta-se às necessidades do sujeito. Além disso, as intervenções baseadas na internet deslocam o foco da doença mental para o comportamento de buscar ajuda. $\mathrm{O}$ indivíduo passa a ser agente de sua própria mudança (Miclea, Miclea, Ciuca, \& Budau, 2010), imprime seu ritmo ao tratamento e pode revisar o material terapêutico sempre que desejar (Proudfoot et al., 2011)

\section{A relação terapêutica na psicoterapia pela internet}

Como se sabe, a relação terapêutica caracteriza-se pelo padrão comunicativo que se estabelece entre terapeuta e paciente na expressão implícita ou explícita de sentimentos e atitudes entre um e outro (Gelso \& Carter, 1985), e tem sido fortemente associada aos resultados do tratamento (Horvath, Del Re, Flückiger, \& Symonds, 2011). É de se esperar que essa relação, na sua forma online, difira qualitativamente de sua versão presencial. No entanto, pesquisas têm apontado semelhanças entre ambas. Escalas de medida da relação terapêutica utilizadas em intervenções psicoterápicas têm mostrado que as pontuações obtidas nas terapias online não diferem significativamente das encontradas nas tradicionais.

Cook e Doyle (2002) compararam a pontuação no Working Alliance Inventory (WAI) de 15 pacientes que receberam psicoterapia online via e-mail, chat ou audioconferência com dados normativos de amostra representativa de terapia presencial de 25 pacientes. O grupo online apresentou maiores médias no escore composto e na subescala objetivos do WAI. Bouchard et al. (2004) observaram pontuação alta nas três subescalas do WAl, desde a primeira sessão, em 21 pacientes que receberam terapia cognitivo-comportamental 
para transtorno do pânico com agorafobia via videoconferência. Os autores não encontraram diferenças significativas quando os resultados foram comparados com os de um grupo de pacientes que recebeu a mesma terapia na modalidade presencial. Prado e Meyer (2006) utilizaram o WAI em 29 pacientes que realizaram psicoterapia pela internet via e-mail, e encontraram relação terapêutica semelhante à descrita na literatura sobre o instrumento. Reynolds, Styles e Grohol (2006) compararam a pontuação no Agnew Relationship Measure (ARM) de 17 pacientes que receberam terapia online via e-mail com dados da literatura acerca da terapia presencial. Os autores encontraram resultados semelhantes em ambos os grupos, sendo que os terapeutas do grupo online apresentaram médias mais altas na subescala confiança. Germain, Marchand, Bouchard, Guay e Drouin (2010) compararam a pontuação no WAI de 17 pacientes que receberam terapia cognitivocomportamental para estresse pós-traumático via videoconferência com 29 pacientes que receberam a mesma terapia na versão presencial, e não encontraram diferenças significativas entre os dois grupos. Preschl, Maercker e Wagner (2011) compararam a pontuação no WAI de 25 pacientes que realizaram terapia cognitivo-comportamental para depressão via videoconferência com 28 pacientes que realizaram a mesma terapia em versão presencial e também não encontraram diferenças significativas entre os dois grupos.

Estudo comparativo (Day \& Schneider, 2002) de três modalidades de terapia (face a face, videoconferência e audioconferência), com 80 pacientes, mostrou que os dois grupos online pontuaram mais na Vanderbilt Psychotherapy Process Scale na dimensão participação do cliente (nível de atividade, iniciativa, confiança, espontaneidade e desinibição) do que o grupo presencial. Uma interpretação possível para os resultados seria a de que os pacientes teriam se esforçado mais para se comunicar por meio da tecnologia digital, tomando para si a responsabilidade da interação. Também a distância poderia ter tornado o autodesvelamento mais seguro.

A sensibilidade experiencial de estudos qualitativos anotou oscilações comunicacionais que requerem atenção na relação terapêutica online. Tratamento por videoconferência apresentou maior dificuldade para terapeutas interpretarem a linguagem corporal dos pacientes, fazerem contato olho no olho e estabelecerem relação empática (Mitchell, Meyers, Swan-Kremeier, \& Wonderlich, 2003). Por sua vez, pacientes relataram que, ao se ajustar ao vídeo, a relação com o terapeuta diferiu das relações face a face, mas não necessariamente de modo pior ou melhor (Simpson, Bell, Knox, \& Mitchell, 2005). Em outro estudo (Himle et al., 2006), os pacientes relataram terem se acomodado rapidamente ao vídeo, sentindo-se na sala do terapeuta.

Resultados similares foram encontrados por Fletcher-Tomenius e Vossler (2009) em análise de entrevistas sobre terapia online via MSN. Quanto ao anonimato, os terapeutas experimentaram um alto nível de confiança e perceberam que seus pacientes desenvolveram-na mais rapidamente do que os da terapia presencial, acreditando que tenham dado um salto de confiança por não terem tantas pistas para confiar no terapeuta. Também os terapeutas tiveram que acreditar em suas representações internas dos pacientes. Os pacientes mostraram-se mais desinibidos do que os da terapia presencial, expondo mais rapidamente seus problemas. As conclusões de Fletcher-Tomenius e Vossler (2009) sintetizam o estado da arte em psicoterapia online por; MSN: 1) recomendada para pessoas que têm medo de estigma e dificuldade em falar de seus problemas, 2) proporciona relação simétrica com o paciente por não haver pistas raciais e étnicas, 3) dá maior controle da situação ao paciente e 4) alcança nível de confiabilidade interpessoal semelhante à terapia tradicional. 
São necessários mais estudos para se avaliar e compreender a relação terapêutica nas diferentes formas de psicoterapia pela internet. No entanto, a literatura vem crescendo nesse sentido, trazendo resultados convergentes, como mostram, por exemplo, os estudos de Hanley, que entrevistou adolescentes que receberam atendimento psicoterápico via e-mail em um site de terapia do Reino Unido, e de Jøraas, Rimehaug, Birkeland e Arefjord (2009), que entrevistaram três terapeutas que proveram follow-up terapêutico online via e-mail a três pacientes adolescentes que terminaram o atendimento presencial. Pode-se concluir que os estudos realizados até agora, apesar de indicarem diferenças e mesmo fraquezas em relação ao atendimento online, reconhecem o campo como promissor para o avanço do atendimento e para a proteção à saúde mental.

\section{Efetividade da psicoterapia pela internet}

Os resultados dos estudos dedicados à efetividade parecem promissores para os atendimentos online. Barak et al. (2008) realizaram uma metanálise com 9.764 pacientes com diferentes transtornos, que receberam diversas formas de intervenção psicológica através da internet, cuja efetividade era avaliada através de diferentes medidas. Os resultados obtidos foram similares aos encontrados na terapia tradicional, mesmo quando efeitos de interação como tipo de terapia online (se automonitorada ou provida por um profissional), tipo de medida de resultados, tempo de medida de resultados (pós-terapia ou follow-up), tipo de transtorno, abordagem terapêutica e modalidade de comunicação. Os autores sugeriram a adoção de intervenções psicológicas online como uma modalidade terapêutica legítima. Também Miclea et al., em uma revisão da literatura, encontraram dados que sugerem que a psicoterapia mediada por computador tem efetividade comparável à das terapias presenciais.

Psicoterapias de diferentes abordagens vêm sendo praticadas online, como psicodinâmica, narrativa, cognitivo-comportamental, comportamental e terapia centrada no cliente. Todavia, não se sabe como essas abordagens são utilizadas ou modificadas no ambiente virtual (Finn \& Barak, 2010). A maior parte dos estudos investiga a efetividade de terapias cognitivo-comportamentais (TCC) online. Elas têm se mostrado efetivas no tratamento de depressão, ansiedade, transtorno do pânico, fobia, e estresse pós-traumático (Cartreine et al., 2010).

Estudos que comparam TCCs online com TCCs presenciais mostram que a modalidade online pode ter resultados semelhantes aos da presencial. Um exemplo é a metanálise de Cuijpers, Donker, van Straten e Andersson (2009), em que compararam resultados de TCCs online com os de TCCs presenciais, e não encontraram diferenças significativas entre ambos. Também quando TCCs individuais online são comparadas com TCCs de grupo presenciais podem apresentar resultados semelhantes (Bergström et al., 2010; Hedman et al., 2011). E ainda TCCs de grupo online e TCCs de grupo presenciais podem apresentar resultados similares (Greene et al., 2010; Morland, Heynes, Mackintosh, Resik, \& Chard, 2011). Igualmente, TCCs online comparadas com outras terapias presenciais podem apresentar resultados superiores, como no estudo de Kessler et al. (2009). Os autores observaram que uma intervenção de TCC online padronizada e praticada por terapeutas treinados apresentou resultados melhores para tratar depressão do que as terapias presenciais oferecidas em postos de saúde do Reino Unido.

TCCs online podem mostrar-se mais efetivas quando comparadas a outras psicoterapias 
Atendimentos via videoconferência

(Skype) têm sido realizados por psicanalistas estrangeiros com mais de 70 chineses em formação na China American Psychoanalytic Alliance (Fishkin, Fishkin, Leli, Katz, \& Snyder, 2011). pela internet. Litz, Engel, Bryant e Papa (2007) estudaram a TCC para estresse pós-traumático automonitorada online, comparando-a com a psicoterapia de apoio online e constataram resultados melhores na primeira. Os terapeutas do grupo experimental, treinados de forma padronizada, encontravamse com os pacientes e planejavam o tratamento adequado. Posteriormente, os pacientes seguiam sozinhos tratamento computadorizado. Os resultados são favoráveis à ampliação da oferta dessa psicoterapia para a população que sofre de estresse pós-traumático nos Estados Unidos, e o Department of Veteran Affairs tem incentivado pesquisas no campo (Gros, Yoder, Tuerk, Lozano, \& Acierno, 2011).

Atendimentos via videoconferência (Skype) têm sido realizados por psicanalistas estrangeiros com mais de 70 chineses em formação na China American Psychoanalytic Alliance (Fishkin, Fishkin, Leli, Katz, \& Snyder, 2011). A prática tem sido percebida pelos analistas como promissora. Segundo um deles, os parâmetros não diferem significativamente daqueles do setting tradicional. Alterações do processo devemse não à utilização do Skype, que considera neutro ao fenômeno, mas a variações de vocabulário, língua, educação, fatores micro e macroculturais. Apesar dessa afirmação, faltam pesquisas que identifiquem como o uso do Skype pode influenciar a efetividade de tratamentos com orientação psicanalítica.

A descrição de como o processo psicanalítico corre em atendimento online oferecido por Fishkin et al. merece destaque. Os analistas prestam atenção ao ritmo da fala do paciente, à espontaneidade ou às interrupções e às qualidades tonais, e identificam a contratransferência escutando seus próprios sentimentos, pensamentos e fantasias, como na análise tradicional. Há, todavia, aspectos novos no tratamento virtual, principalmente, as muitas pistas para a desconcentração de terapeuta ou paciente. Por exemplo, o atendimento pode ocorrer por laptop, em um parque ou em um shopping, ou o terapeuta pode distrair-se com as muitas mensagens que passam pela tela do computador.

Estudos indicam que o grau de envolvimento com o terapeuta se relaciona com os resultados das intervenções online. Diferentes graus de interação entre terapeuta e paciente são previstos nas mais diversas formas de psicoterapia pela internet, e busca-se conhecer melhor seus efeitos. Preschl et al. (2011) revisaram estudos que compararam resultados de diferentes terapias baseadas na internet para depressão. Os autores concluíram que um mínimo de contato com o terapeuta é necessário para reduzir o abandono de terapia e para aliviar os sintomas. As terapias online automonitoradas apresentaram maior índice de abandono e efeitos reduzidos em comparação com as que envolveram interação com o terapeuta. Andersson e Cuijpers (2009) realizaram uma metanálise de intervenções baseadas na internet para depressão e encontraram uma forte influência do apoio do terapeuta nos resultados. Seria suficiente o terapeuta dispender 100 minutos por paciente em programas de dez semanas, comentando as tarefas realizadas e provendo feedback (Andersson, Carlbring, Berger, Almlov, \& Cuijpers 2009). Aumentar o contato com o terapeuta além do necessário parece não influenciar os ganhos terapêuticos (Vernmark et at., 2010).

Berger et al. (2011) contrariaram achados de que o grau de interação com o terapeuta influi nos resultados. Em ensaio clínico randomizado com 81 pacientes com fobia social, os autores observaram resultados semelhantes em três modalidades de terapia cognitivo-comportamental online: 1) terapia automonitorada, 2) terapia com auxílio de um terapeuta via e-mail uma vez por semana e 3) terapia com apoio de um terapeuta 
por e-mail ou telefone na frequência que o paciente necessitou. Klein et al. (2009) também encontraram resultados similares quando 57 pacientes foram divididos em duas intervenções baseadas na internet para transtorno do pânico, que diferiram apenas na frequência de apoio do terapeuta: se de três e-mails por semana ou de um e-mail por semana. Titov (2011), nesse mesmo sentido, sugeriu que tratamentos baseados na internet altamente estruturados com pouca interação com o terapeuta podem atingir excelentes resultados.

Apesar do crescente interesse na influência do apoio do terapeuta nas intervenções baseadas na internet, há pouca pesquisa sobre os fatores de processo e preditores de resultados nesses tratamentos. Não se sabe se os fatores e os processos terapêuticos responsáveis pela redução de sintomas na terapia tradicional operam da mesma forma nesses programas computadorizados (Preschl et al., 2011).

\section{Questões legais e éticas da psicoterapia pela internet}

Para Finn e Barak (2010), a psicoterapia pela internet deve seguir as mesmas normas do código de ética profissional para atendimento face a face, como confidencialidade, disponibilidade em caso de emergência, intervenção em situações em que o paciente apresenta risco a si próprio ou a outros e delação de abuso de menor e cumprimento da legislação local quanto à licença para atuar. Nos EUA, por exemplo, há decisões judiciais que entendem que as normas que incidem são as da localidade do paciente, e alguns Estados impedem que seus cidadãos contratem serviços de terapeutas sem licença local.

Na psicoterapia online, existem apreensões quanto à capacidade de os pacientes proverem sua real identidade, quanto à falta de segurança da internet na privacidade e na confidencialidade, quanto à fragilidade de se depender da tecnologia eletrônica e quanto à assistência em casos de emergência. Também causa preocupação a dificuldade de, na internet, os terapeutas comunicarem mensagens precisas, manifestarem sentimentos, lidarem com diferenças culturais e resolverem pagamento de serviços. Barnett (2011) considera que o profissional deve informar o paciente dos riscos e dos limites da terapia pela internet e dos requisitos tecnológicos necessários para a participação online. O terapeuta deve combinar como serão solucionadas as falhas na comunicação eletrônica (se imediatamente contatará o paciente por telefone) e como será o manejo em casos de emergência, incluindo acordo sobre recursos na área do paciente que poderão ser acionados. Também deve ser especificado como e de que forma (se via e-mail, ou mensagem texto no celular, ou telefone, etc.) será o acesso ao terapeuta entre as sessões, para que o paciente não tenha expectativas irreais quanto a sua disponibilidade. Igualmente deve ser informado ao paciente que tipo de contato incidental é cobrado. A competência do terapeuta quanto à tecnologia utilizada é fundamental, conforme o Código de Ética da American Psychological Association, incluindo treino e supervisão. Ela habilita o profissional a lidar com dificuldades e falhas na tecnologia bem como a prover instruções ao paciente para seu uso apropriado. Para minimizar riscos, as organizações dos profissionais que praticam terapia online desenvolveram diretrizes. Não se sabe, todavia, em que medida são seguidas. Yazvac (2009) observou que sites de terapeutas nos Estados Unidos 
mostram pouco comprometimento ético. Apesar disso, a busca de serviços em sites de terapia tem crescido de forma consistente, mesmo que não sigam diretrizes profissionais e provejam informação limitada sobre sua política e procedimentos.

Finn e Barak (2010) observaram, em uma survey na internet com 93 terapeutas online, que menos da metade considerou que sua organização profissional tinha diretrizes claras sobre a prática. A maior parte não julgou importante confirmar a identidade do cliente e considerou que a terapia online se prestava a assuntos interpessoais e sociais, mas não a situações de risco de suicídio, violência doméstica, abuso de substâncias, abuso infantil ou estupro. Dentre os que detectaram casos a serem avisados às autoridades $(\mathrm{N}=13), 42 \%$ o fizeram. Um quarto dos participantes se deparou com situação em que o paciente apresentava risco a si mesmo ou a outros, mas menos da metade avisou as autoridades. Quanto aos terapeutas que atuavam nos Estados Unidos $(\mathrm{N}=76)$, mais da metade informou não levar em consideração aspectos jurisdicionais, somente $5 \%$ restringindo seus atendimentos a pacientes do mesmo estado. Do total de terapeutas que participaram da enquete, a maior parte não realizou treinamento em psicoterapia pela internet, nem recebeu supervisão. Os autores concluíram que há falta de consenso desses profissionais quanto a suas obrigações legais e éticas, e que seria necessário um treino formal em psicoterapia online.

Também na utilização de programas computadorizados de terapia devem ser observadas questões legais e éticas, provendose informações relevantes ao paciente para que este possa escolher se deseja ou não receber a intervenção. O paciente deve ser alertado dos termos e das condições do programa, de seus direitos e responsabilidades bem como dos riscos e benefícios da intervenção (Proudfoot et al., 2011).

\section{Conclusão}

A psicoterapia online já se desenvolve no exterior há mais de dez anos, com resultados bastante promissores. A compilação de achados na área constitui forte evidência a seu favor, o que não pode ser ignorado em debates sensatos acerca do tema. Estudos têm mostrado que a utilização da internet para atendimento psicoterápico pode não causar tanto prejuízo à relação terapêutica quanto se pensa. Também pesquisas acerca da efetividade de tratamentos online, mesmo sem a participação de terapeutas, têm apresentado resultados positivos. As questões legais e éticas que se delineiam no campo parecem ser parcialmente resolvidas com diretrizes claras para a prática e o treino.

Todavia, algumas questões carecem de respostas, como que tipo de paciente pode beneficiar-se da terapia online e qual forma de tratamento pode oferecer melhores resultados para determinada demanda. São necessários mais estudos para que se compreendam as peculiaridades da psicoterapia pela internet. Um exame aprofundado pode auxiliar-nos a compreender como as diferentes abordagens psicoterápicas são utilizadas ou modificadas no ambiente virtual e quais requisitos são necessários para que sua prática ocorra em benefício do paciente.

Talvez os esforços devam voltar-se não para a proibição da psicoterapia online, mas sim, para sua efetiva regulamentação, a qual pode dar-se, em grande medida, a partir da experiência dos profissionais e 
da implementação de programas na rede pública. Alguns países têm procedido dessa forma, fazendo o caminho ao percorrê-lo.

Também as investigações em psicoterapia online já estão bastante avançadas no exterior, enquanto, no Brasil, nós nos limitamos a discutir o tema. A psicoterapia pela internet pode ser uma área promissora para pesquisas nacionais, já que nos seria muito útil devido ao extenso território de nosso país, ao grande número de filas de espera para atendimento psicoterápico e à falta de conhecimentos em saúde mental por parte da população. Ela permitiria ampliar o acesso à terapia, prover recursos aos que aguardam tratamento e melhorar os conhecimentos em saúde mental de nosso povo. 


\section{Maria Adélia Minghelli Pieta}

Doutoranda em Psicologia na Universidade Federal do Rio Grande do Sul, Porto Alegre - RS - Brasil.

E-mail: mampieta@gmail.com

\section{William B. Gomes}

Doutor em Higher Education pela Southern Illinois University Carbondale e Professor Adjunto da Universidade Federal do Rio Grande do Sul, Porto Alegre - RS - Brasil.

E-mail: gomesw@ufrgs.br

Endereço para envio de correspondência:

Rua Ramiro Barcelos, 2600. CEP: 90035-003. Porto Alegre, RS.

Recebido 07/05/2012, 1a Reformulação 19/04/2013, Aprovado 29/08/2013. 


\section{Referências}

Andersson, G., \& Cuijpers, P. (2009). Internet-based and other computerized psychological treatments for adult depression: A meta-analysis. Cognitive Behavioural Therapy, 38(1) 196-205. doi: 10.1080/16506070903318960

Andersson, G., Carlbring, P., Berger, T., Almlov, J., \& Cuijpers, P. (2009). What makes internet therapy work? Cognitive Behavavioural Therapy, 38(1), doi: 55-60. 10.1080/16506070902916400

Barak, A., Hen, L., Boniel-Nissim, M., \& Shapira, N. (2008). A comprehensive review and a meta-analysis of the effectiveness of internet-based psychotherapeutic interventions. Journal of Technology in Human Services, 26(2/4), 109-160. doi : $10.1080 / 15228830802094429$

Barak. A, Klein, B., \& Proudfoot, J. G. (2009). Defining internetsupported therapeutic interventions. Annals of Behavioral Medicine, 38(1), 4-17. doi: 10.1007/s12160-009-9130-7

Barnett, J. E. (2011). Utilizing technological innovations to enhance psychotherapy supervision, training, and outcomes. Psychotherapy, 48(2), 103-108. doi: 10.1037/a0023381

Berger, T., Caspar, F., Richardson, R., Kneubühler, B., Sutter, D., \& Andersson, G. (2011). Internet-based treatment of social phobia: A randomized controlled trial comparing unguided with two types of guided self-help. Behaviour Research and Therapy, 49(3), 158-169. doi: 10.1016/j.brat.2010.12.007

Bergström, J., Andersson, G., Ljótsson, B., Rück, C., Andréewitch, S., Karlsson, A. et al. (2010). Internet versus groupadministered cognitive behaviour therapy for panic disorder in a psychiatric setting: A randomised trial. BMC Psychiatry, 10(54), 1-10. doi: 10.1186/1471-244X-10-54.

Bouchard, S., Paquin, B., Payeur, R., Allard, M., Rivard, V., Fournier, T., Renaud, P., \& Lapierre, J. (2004). Delivering cognitive-behavior therapy for panic disorder with agoraphobia in videoconference. Telemedicine Journal and E-health, 10(1), 13-25. doi:10.1089/153056204773644535

Carrad, I., Crépin, C., Rouget, P., Lam, T., Golay, A., \& Van der Linden, M. (2011). Randomised controlled trial of a guided self-help treatment on the internet for binge eating disorder. Behaviour Research and Therapy, 49(8), 482-491. doi: 10.1016/j.brat.2011.05.004.

Cartreine, J. A., Ahern, D. K., \& Locke, S. E. (2010). A roadmap to computer-based psychotherapy in the United States. Harvard Review of Psychiatry, 18(2), 80-95. doi: 10.3109/10673221003707702.

Cluver, J. S., Schuyler, D., Frueh, B. C., Brescia, F., \& Arana, G. W. (2005). Remote psychotherapy for terminally ill cancer patients. Journal of Telemedicine and Telecare, 11(3), 157-159. doi: $10.1258 / 1357633053688741$

Conselho Federal de Psicologia. (2005). Regulamenta o atendimento psicoterapêutico e outros serviços psicológicos mediados por computador e revoga a Resolução CFP no003/2000. Resolução CFP n ${ }^{\circ}$ 012/2005. Brasília, DF: Autor

Cook, J. E., \& Doyle, C. (2002). Working alliance in online therapy as compared to face-to-face therapy: Preliminary results. CyberPsychology \& Behavior, 5(2), 95-105. doi:10.1089/109493102753770480

Cuijpers, P., Marks, I. M., van Straten, A., Cavanagh, K., Gega, L., \& Andersson, G. (2009). Computer-aided psychotherapy for anxiety disorders: A meta-analytic review. Cognitive Behaviour Therapy, 38(2), 66-82. doi: 10.1080/16506070802694776
Day, S., \& Schneider, P. (2002). Psychotherapy using distance technology: A comparison of face-to-face, video, and audio treatment. Journal of Counseling Psychology, 49(4), 499-503. doi:10.1037//0022-0167.49.4.499

Finn, J., \& Barak, A. (2010). A descriptive study of e-counsellor attitudes, ethics, and practice. Counselling and Psychotherapy Research: Linking research with Practice, 10(4), 268-277. doi: 10.1080/14733140903380847

Fishkin, R., Fishkin, L., Leli, U., Katz, B., \& Snyder, E. (2011). Psychodynamic treatment, training, and supervision using internet-based technologies. Journal of the American Academy of Psychoanalysis and Dynamic Psychiatry, 39(1) 155-168. doi: 10.1521/jaap.2011.39.1.155.

Fletcher-Tomenius, L., \& Vossler, A. (2009). Trust in online therapeutic relationships: The therapist's experience. Counselling Psychology Review, 24(2), 24-34.

Gaisbury, S., \& Blaszczynski, A. (2011). A systematic review of internet-based therapy for the treatment of addictions. Clinical Psychology Review, 31(3), 490-498. doi: 10.1016/j. cpr.2010.11.007

Gelso, C. J., \& Carter, J. A. (1985). The relationship in counseling and psychotherapy: Components, consequences, and theoretical antecedents. The Counseling Psychologist, 13(2), 155-243. doi: 10.1177/0011000085132001

Germain, V., Marchand, A., Bouchard, S., Guay, S., \& Drouin, M. (2010). Assessment of the therapeutic alliance in face-toface or videoconference treatment for posttraumatic stress disorder. Cyberpsychology, Behavior, and Social Networking, 13(1), 29-35. doi:10.1089/cyber.2009.0139.

Godleski, L., Nieves, E., Darkins, A., \& Lehmann, L. (2008). VA telelemental health: Suicide assessment. Behavioral Sciences and the Law, 26, 271-286. doi: 10.1002/bsl.811

Greene, C. J., Morland, L. A., Macdonald, A., Frueh, B. C., Grubbs, K. M., \& Rosen, C. S. (2010). How does tele-mental health affect group therapy process? Secondary analysis of a noninferiority trial. Journal of Consulting and Clinical Psychology, 78(5), 746-750. doi: 10.1037/a0020158

Gros, D. F., Yoder, M., Tuerk, P. W., Lozano, B. E., \& Acierno, R. (2011). Exposure therapy for PTSD delivered to veterans via telehealth: Predictors of treatment completion and outcome and comparison to treatment delivered in person. Behavior Therapy, 42(2), 276-283. doi:http://dx.doi.org/10.1016/j. beth.2010.07.005

Hanley, T. (2009). The working alliance in online therapy with young people: Preliminary findings. British Journal of Guidance \& Counselling, 37(3), 257-269. doi: $10.1080 / 03069880902956991$

Hedman, E., Andersson, G., Ljótsson, B., Andersson, E., Rück C., et al. (2011). Internet-based cognitive behavior therapy vs. cognitive behavioral group therapy for social anxiety disorder: A randomized controlled non-inferiority trial. PLoS ONE, 6(3), e18001. doi: 10.1371/journal.pone.0018001

Himle, J. A., Fischer, D. J., Muroff, J. R., Van Etten, M. L., Lokers, L. M., Abelson, J. L., \& Hanna, G. L. (2006). Videoconferencingbased cognitive-behavioral therapy for obsessive-compulsive disorder. Behaviour Research and Therapy, 44(12), 1821-1829. doi: http://dx.doi.org/10.1016/j.brat.2005.12.010

Horvath, A. O., Del Re, A. C., Flückiger, C., \& Symonds, D. (2011) Alliance in individual psychotherapy. Psychotherapy: Theory, Research, Practice, Training, 48(1), 9-16. doi: 10.1037/ a0022186 
Jøraas, T., Rimehaug, T., Birkeland, M. S., \& Arefjord, K. (2009). E-therapy as a follow-up supplement to outpatient treatment of adolescents: A qualitative study of therapist experiences. Tidsskrift for Norsk Psykologforening, 46(4), 355-361.

Kessler, D., Lewis, G., Kaur, S., Wiles, N., King, M., Weich, S., \& Sharp, D. J. et al. (2009). Therapist-delivered internet psychotherapy for depression in primary care: A randomised controlled trial. Lancet, 374, 628-634. doi:10.1016/S01406736(09)61257-5

King, R., Bambling, M., Lloyd, C., Gomurra, R., Smith, S., Reid, W., \& Wegner, K. (2006). Online counselling: The motives and experiences of young people who choose the internet instead of face to face or telephone counseling. Counselling and Psychotherapy Research, 6(3), 169-174.doi: 10.1080/14733140600848179

Klein, B., Austin, D., Pier, C., Kiropoulos, L., Shandley, K., \& Mithcell, J. et al. (2009). Internet-based treatment for panic disorder: Does frequency of therapist contact make a difference? Cognitive Behaviour Therapy, 38(2), 100-113. doi 10.1080/16506070802561132

Klein, B., Mitchell, J., Abbott, J., Shandley, K., Austin, D., \& Gilson, K. et al. (2010). A therapist-assisted cognitive behavior therapy internet intervention for posttraumatic stress disorder: Pre-, post- and 3-month follow-up results from an open trial. Journal of Anxiety Disorders, 24, 635-644. doi: http://dx.doi. org/10.1016/j.janxdis.2010.04.005

Leibert, T., Archer Jr., J., Munson, J., \& York, G. (2006). An exploratory study of client perceptions of internet counseling and the therapeutic alliance. Journal of Mental Health Counseling, 28(1), 69-83.

Litz, B. T., Engel, C. C., Bryant, R. A., \& Papa, A. (2007). A randomized, controlled proof-of-concept trial of an internetbased, therapist-assisted self-management treatment for posttraumatic stress disorder. American Journal of Psychiatry, 164(11), 1676-1683. doi:10.1176/appi.ajp.2007.06122057

Macdonald, W., Mead, N., Bower, P., Richards, D., \& Lovell, K. (2007). A qualitative study of patients' perceptions of a 'minimal' psychological therapy. International Journal of Social Psychiatry, 53, 23-35. doi: 10.1177/0020764006066841

MacGregor, A., Hayward, L., Peck, D. F., \& Wilkes, P. (2009). Empirically grounded clinical interventions: Client's and refereers' perceptions of computer-guided CBT (FearFighter). Behavioural and Cognitive Psychotherapy, 37, 1-9.

March, S., Spence, S. H., \& Donovan, C. L. (2009). The efficacy of an internet-based cognitive-behavioral therapy intervention for child anxiety disorders. Journal of Pediatric Psychology, 34(5), 474-487. doi:10.1093/jpepsy/jsn099

Miclea, M., Miclea, S., Ciuca, A. M., \& Budau, O. (2010). Computer-mediated psychotherapy. Present and prospects. A developer perspective. Cognition, Brain, Behavior: An Interdisciplinary Journal, 14(3), 185-208.

Mitchell, J. E., Meyers, T., Swan-Kremeier, L., \& Wonderlich, S. (2003). Psychotherapy for bulimia nervosa delivered via telemedicine. European Eating Disorders Review, 11(3), 222230. doi: 10.1002/erv.517

Moore, D., Guthmann, D., Rogers, N., Frakeer, S., \& Embree, J. (2009). E-therapy as a means for addressing barriers to substance use disorder treatment for persons who are deaf. Journal of Sociology \& Social Welfare, 36(4), 75-92.

Morland, L. A., Hynes, A. K., Mackintosh, M. A., Resick, P. A., \& Chard, K. M. (2011). Group cognitive processing therapy delivered to veterans via telehealth: A pilot cohort. Journal of Traumatic Stress, 24(4), 465-469. doi: 10.1002/jts.20661

Mucic, D. (2010). Transcultural telepsychiatry and its impact on patient satisfaction. Journal of Telemedicine and Telecare, 16(5), 237-242. doi: 10.1258/jtt.2009.090811.

Prado, O. Z., \& Meyer, S. B. (2006). Avaliação da relação terapêutica na terapia assíncrona via internet. Psicologia em Estudo, 11(2), 247-257. doi: http://dx.doi.org/10.1590/S141373722006000200003

Preschl, B., Maercker, A., \& Wagner, B. (2011). The working alliance in a randomized controlled trial comparing online with face-to-face cognitive-behavioral therapy for depression. BMC Psychiatry, 11(189), 2-10. doi:10.1186/1471-244X-11-189

Proudfoot, J., Klein, B., Barak, A., Carlbring, P., Cuijpers, P., Lange, A., Ritterband, L., \& Andersson, G. (2011). Establishing guidelines for executing and reporting internet intervention research. Cognitive Behaviour Therapy, 40(2), 82-97. doi: http://dx.doi.org/10.1080/16506073.2011.573807

Reynolds, D. J., Stiles, W. B., \& Grohol, J. (2006). An investigation of session impact and alliance in internet based psychotherapy: Preliminary results. Counselling and Psychotherapy Research, 6(3), 164-168. doi: 10.1080/14733140600853617

Rochlen, A. B., Zack, J. S., Speyer, C. (2004). Online therapy: Review of relevant definitions, debates, and current empirical support. Journal of Clinical Psychology, 60(3), 269-283. doi: $10.1002 /$ jclp. 10263

Scharff, J. F. (2012). Clinical issues in analyses over the telephone and the internet. International Journal of Psychoanalysis, 93, 81-95. doi: 10.1111/j.1745-8315.2011.00548.x

Sharp, I. R., Kobak, K. A., \& Osman, D. A. (2011). The use of videoconferencing with patients with psychosis: A review of the literature. Annals of General Psychiatry, 10(1), 14. doi: 10.1186/1744-859X-10-14

Simpson, S., Bell, L., Knox, J., \& Mitchell, D. (2005). Therapy via videoconferencing: A route to client empowerment? Clinical Psychology \& Psychotherapy, 12(2), 156-165. doi: 10.1002/ cpp. 436

Spence, S. H., Donovan, C. L., March, S., Gamble, A., Anderson, R. E., Prosser, S., \& Kenardy, J. (2011). A randomized controlled trial of online versus clinic-based CBT for adolescent anxiety. Journal of Consulting and Clinical Psychology, 79(5), 629-642. doi: $10.1037 / \mathrm{a} 0024512$

Titov, N. (2011). Internet-delivered psychotherapy for depression in adults. Current Opinion in Psychiatry, 24(1), 18-23. doi: 10.1097/YCO.0b013e32833ed18f

Titov, N., Dear, B. F., Schwencke, G., Andrews, G., Johnston, L., Craske, M. G., \& McEvoy, P. (2011). Transdiagnostic internet treatment for anxiety and depression: A randomized controlled trial. Behaviour Research and Therapy, 49(8), 441-452. doi: http://dx.doi.org/10.1016/j.brat.2011.03.007

Yazvac, J. (2009). Website compliance with ethical guidelines by psychologists and professional counselors. Dissertation Abstracts International: Section $B$ : The Sciences and Engineering, 70(1), 9.

Vernmark, K., Lenndin, J., Carlsson, M., Öberg, J., Carlbring, P., Eriksson, T., \& Andersson, G. (2010). Internet administered guided selfhelp versus individualized e-mail therapy: A randomized trial of two versions of CBT for major depression. Behaviour Research and Therapy, 48, 368-376. doi: 10.1016/j. brat.2010.01.005 